\title{
Spinal Cord Tethering, a Very Rare Cause of Cauda Equina Syndrome after Lumbar Disk Surgery: A Case Report
}

\author{
Alireza Tabibkhooei ${ }^{1}$, Farid Kazemi ${ }^{1}$, Foad Kazemi ${ }^{1}$, Morteza Taheri ${ }^{2}$ \\ ${ }^{1}$ Department of Neurosurgery, Iran University of Medical Sciences, Rasool Akram \\ Hospital, Tehran, Iran; \\ ${ }^{2}$ Department of Neurosurgery, Iran University of Medical Sciences, 7Tir Hospital, \\ Tehran, Iran \\ Received November 30, 2019; Accepted February 17, 2020.
}

Key words: Tethered cord syndrome - Cauda equina syndrome - Lumbar disc Surgical complication

Abstract: Tethered cord syndrome (TCS) may rarely remain asymptomatic until degenerative or nondegenerative lumbar diseases superimpose in adulthood and expose the hidden anomaly. In such cases, different treatment options can be selected and simultaneous detethering might be considered too. We are reporting an undiscovered TCS in a young lady who underwent lumbar diskectomy due to symptomatic disk extrusion and suffered complete cauda equina syndrome (CES), postoperatively.

Mailing Address: Morteza Taheri, MD., Department of Neurosurgery, Iran University of Medical Sciences, 7Tir Hospital, Tehran, Iran; Phones: +989120194 908, +982 166503 890; Fax: +982 166509 120; e-mail: drtaheri38@yahoo.com 


\section{Introduction}

In $95 \%$ of the general population, the spinal cord normally ends above or at the level of inferior aspect L2 vertebral body (Srinivas et al., 2012). Some pathologies such as thickened filum terminale, or a lipomatous lesion cause to the cord ends at the level below than L2 and result in low-lying tethered cord. In childhood, during the growth of the spine, longitudinal traction on the tethered cord can result in a neurological impairment named tethered cord syndrome (TCS). Rarely, the patients remain asymptomatic to adulthood. In some of these patients, precipitating factors such as trauma or spinal degenerative conditions exacerbate neurological symptoms, and patients can present with tethered cord syndrome (Srinivas et al., 2012; Carpineta et al., 2017). On the other hand, TCS is an error of spinal cord developmental process which could show neurological signs and symptoms in childhood or adulthood due to tension on conus medullaris (Lapsiwala and Iskandar, 2004; Yamada et al., 2004).

Injury or irritation of distal lumbosacral roots in cauda equina may lead to a complex syndrome of sensory, motor, and sphincter disturbances which named cauda equina syndrome (CES). CES or horsetail syndrome is a clinically important and troublesome condition and rarely occurs following lumbar spine surgery (Duncan and Bailey, 2011). We are going to report a case of postoperative CES in a patient with a symptomatic extruded disc with undiagnosed TCS. We report the clinical scenario and will also discuss the possible mechanisms of postoperative complications.

\section{Case report}

A 32-years-old healthy woman referred us due to intractable bilateral L5 and S1 radicular pain, mild to moderate low back pain, and feet paresthesia since three months ago. She hadn't any sphincter problems such as frequency, urgency or incontinence. Physical examination revealed positive bilateral Lasegue's sign, no motor or sensory deficit, and no upper motor neuron sign. On magnetic resonance imaging (MRI), we noted a voluminous extruded disk at L4/L5 level with significant compression on thecal sac (Figure 1).

The patient scheduled for lumbar discectomy. During surgery after L4 coronal hemilaminectomy and bilateral foraminotomy, we found bilateral conjoined roots; and discectomy was performed with gentle retraction of exiting roots without any intraoperative complication. Just postoperatively, we faced with a CES for which we didn't have any explanation (except for intraoperative trauma to nerve roots secondary to the aforementioned abnormality). The patient presented with urinary retention and saddle anesthesia without any motor deficit even in S1 myotomes.

On postoperative lumbosacral MRI, thecal sac and neural elements were decompressed, but unexpectedly we detected cord tethering and a low lying conus at L4/L5 level (Figure 2). Reviewing the preoperative images revealed that this 


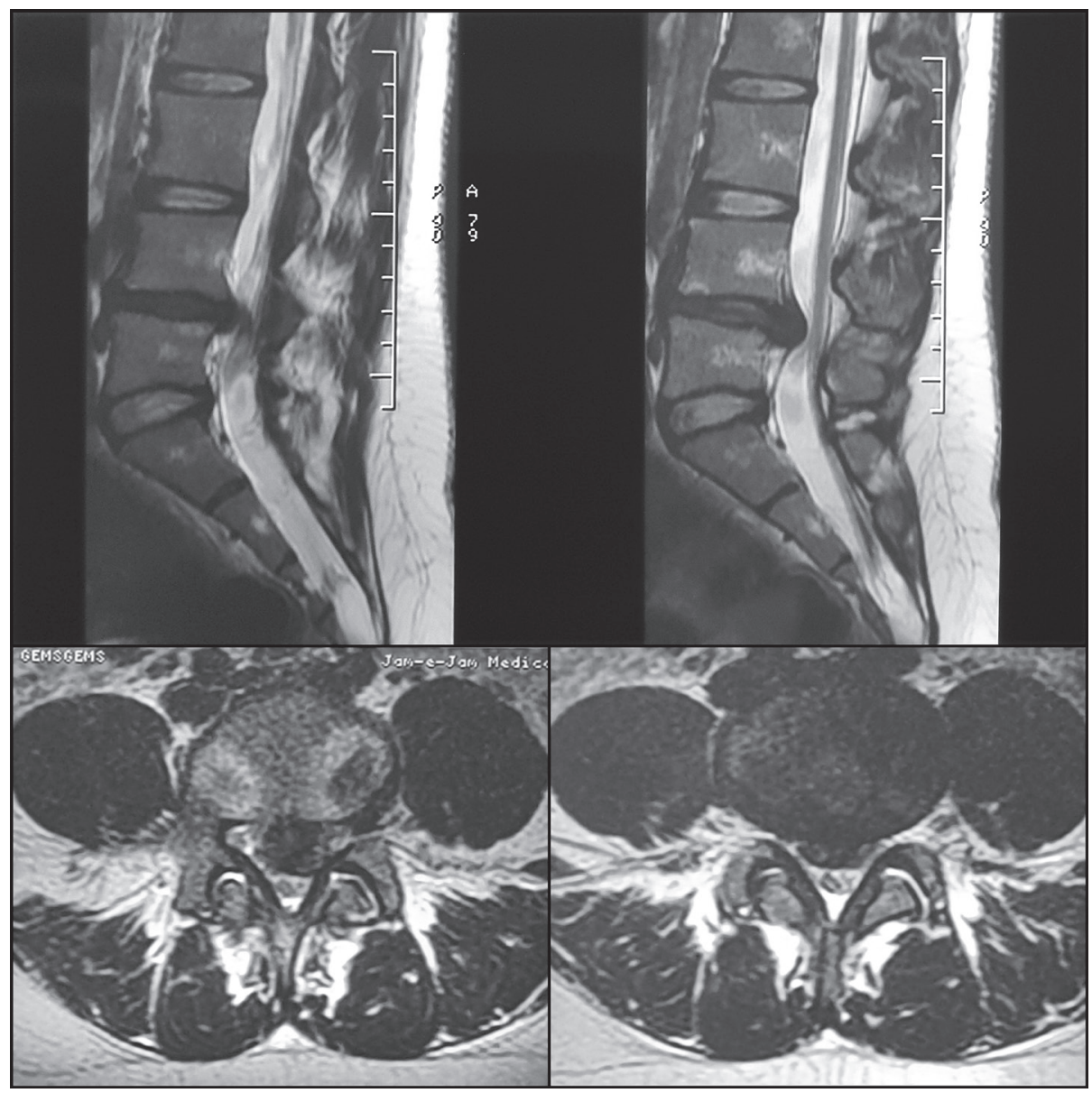

Figure 1 - Preoperative T2 weighted sagittal and axial magnetic resonance imaging of lumbar spine showing bulky disk extrusion at $L 4 / L 5$ level compressing conus medullaris of the tethered cord and exiting roots.

pathology sneaked due to bolded herniated disk. So, a revision surgery for cord untethering offered to the patient but she refused.

Alternative conservative treatments such as steroids, anticholinergics and physical therapies for pelvic floor muscles failed and electrodiagnostic studies showed severe injury to S2 to S4 roots. On the last follow-up, one year after surgery, the patient revealed no significant improvement.

\section{Discussion}

TCS was described the last three decades and was previously known as an only pediatric defect; now, however, there is good evidence on the occurrence of tethered cord in adults and although rare is more common than previously thought 


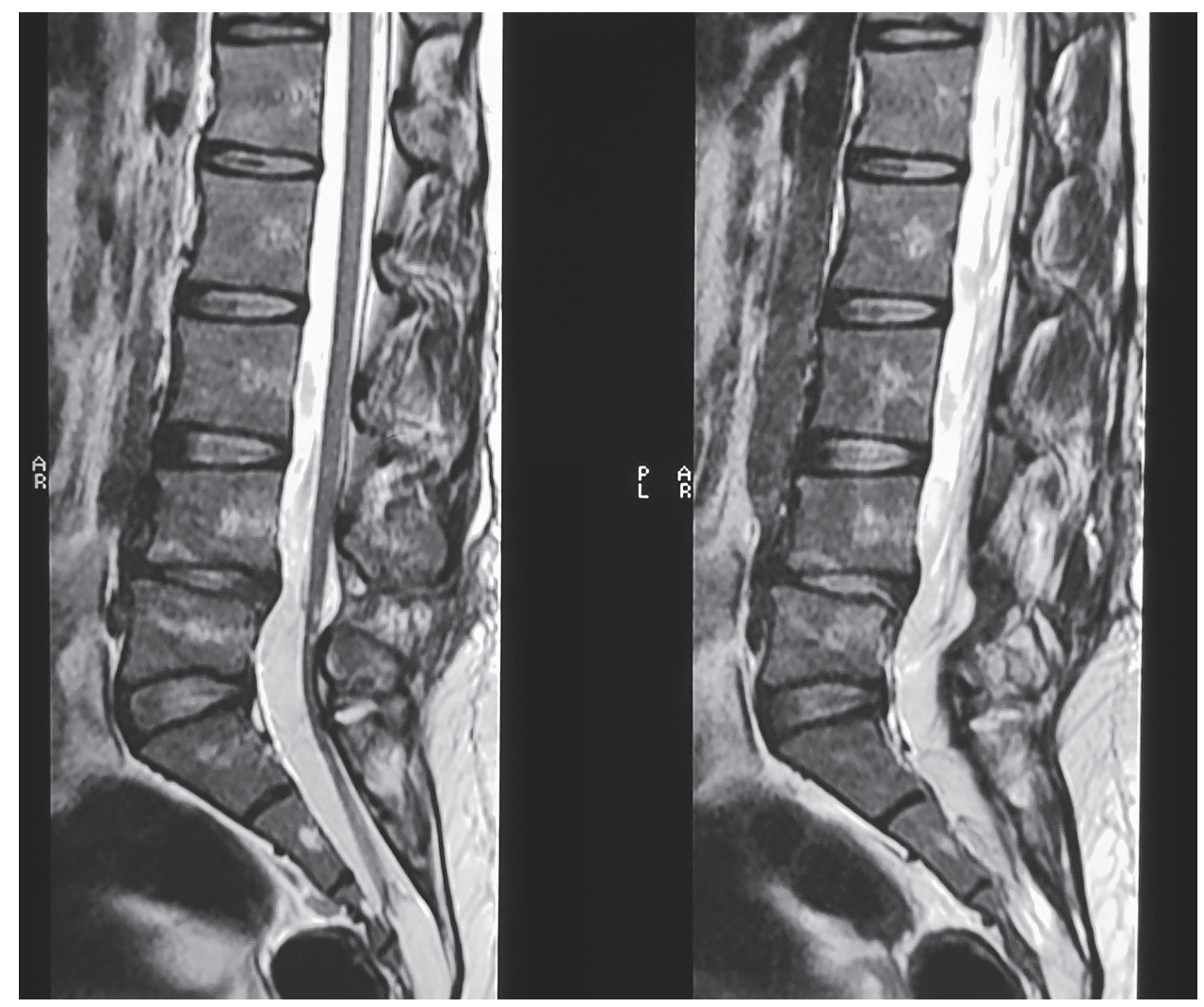

Figure 2 - Postoperative sagittal magnetic resonance imaging revealed tethered cord syndrome and a small area of hypersignality in conus medullaris on T2 weighted lumbar spine images.

(Srinivas et al., 2012). In most of these cases, precipitating factors such as trauma (trauma during surgery could be considered as precipitating factor in our case) or rarely spinal degeneration such as disk diseases, initiate or exacerbate symptoms and deficits (Ahn et al., 2000; Gleave and Macfarlane, 2002; Srinivas et al., 2012). Management of adult-onset tethered cord syndrome is controversial and choosing surgery is debated in literature till now, although the necessity of surgery as the main choice in pediatric cases is well-established (Iskandar et al., 2001; Kang et al., 2003; Aufschnaiter et al., 2008). It has been shown in studies that after a precipitating factor such as coughing, bending, or strenuous physical activity, all as a mechanical longitudinal traction, some symptoms may become apparent that could mimic symptoms of lumbar disc disease (maybe as was seen in our case) or spinal stenosis, but true radicular pain is rare (Srinivas et al., 2012).

The incidence of postoperative cauda equina syndrome is between 0.02 to $1 \%$ in different studies (Henriques et al., 2001). In the majority of cases, the cause is 
retained disk fragment, gelfoam, excessive tension on dural sac during discectomy, epidural abscess, placement of a free epidural fat graft, vascular insufficiency and very rarely dural sac shift and venous congestion (Henriques et al., 2001; Maki et al., 2017). Our case presents postoperative CES as a complication of lumbar discectomy for spinal disc protrusion in a patient with an unknown tethered cord till adulthood. Theoretically, the cause of postoperative CES after that surgery could be the result of tension on sacral nerve roots and the tethered cord. In such cases, motor deficit is almost always present and its return signals a better prognosis (Ahn et al., 2000; Jensen, 2004) in contrast to what happened to our patient.

Reviewing the preoperative MRI sequences demonstrated low lying conus and tethered cord, but the massive disk herniation misled us and obscured coexisting TCS, so the surgical technique was chosen as a routine lower lumbar discectomy procedure. Surely, these special cases should be managed as a thoracic disc herniation. In this case, decompression of neural elements must be performed with minimal traction on the dural sac, for example using transfacet or even anterolateral approaches (Endo et al., 2014; Carpineta et al., 2017). Moreover, intraoperative neuromonitoring could be used before the surgery, if the diagnosis of TCS would be made preoperative. We think it could be helpful for the avoidance of neurological complications; although neuromonitoring findings are not always predictive (Cole et al., 2014).

Although untethering procedure during or after the decompressive surgery in such cases is still in question, especially if patients symptoms could be attributed only to herniated disk and nerve root compression (Endo et al., 2014; Carpineta et al., 2017); it seems that untethering in our case after clarifying underlying disease should be advisable. However, a bright signal on T2 weighted images on postoperative MRI in conus medullaris points to concomitant traumatic contusion within the terminal of cord and poor prognosis for any further treatment (Ramon et al., 1997).

\section{Conclusion}

Although TCS is a rare incidental finding in adult patients, it should be searched in imaging of any patient whose main problem in lumbar spine is a different pathology. In such cases diagnostic and therapeutic measures would be remarkably different too.

\section{References}

Ahn, U. M., Ahn, N. U., Buchowski, J. M., Garrett, E. S., Sieber, A. N., Kostuik, J. P. (2000) Cauda equina syndrome secondary to lumbar disc herniation: a meta-analysis of surgical outcomes. Spine (Phila. Pa. 1976) 25(12), 1515-1522.

Aufschnaiter, K., Fellner, F., Wurm, G. (2008) Surgery in adult onset tethered cord syndrome (ATCS): review of literature on occasion of an exceptional case. Neurosurg. Rev. 31(4), 371-383; discussion 384.

Carpineta, E., Roperto, R., Cacciotti, G., Mastronardi, L. (2017) Tethered spinal cord syndrome with lumbar segmental stenosis treated with XLIF. Interdiscip. Neurosurg. 9, 68-70. 
Cole, T., Veeravagu, A., Zhang, M., Li, A., Ratliff, J. K. (2014) Intraoperative neuromonitoring in single-level spinal procedures: A retrospective propensity score-matched analysis in a national longitudinal database. Spine (Phila. Pa. 1976) 39(23), 1950-1959.

Duncan, J. W., Bailey, R. A. (2011) Cauda equina syndrome following decompression for spinal stenosis. Global Spine J. 1(1), 15-18.

Endo, F., lizuka, H., lizuka, Y., Kobayashi, R., Mieda, T., Takagishi, K. (2014) Myelopathy due to lumbar disc herniation in the presence of a tethered cord. Spinal Cord 52, S11-S13 (Suppl. 1).

Gleave, J. R., Macfarlane, R. (2002) Cauda equina syndrome: What is the relationship between timing of surgery and outcome? Br. J. Neurosurg. 16(4), 325-328.

Henriques, T., Olerud, C., Petren-Mallmin, M., Ahl, T. (2001) Cauda equina syndrome as a postoperative complication in five patients operated for lumbar disc herniation. Spine (Phila. Pa. 1976) 26(3), $293-297$.

Iskandar, B. J., Fulmer, B. B., Hadley, M. N., Oakes, W. J. (2001) Congenital tethered spinal cord syndrome in adults. Neurosurg. Focus 10(1), e7.

Jensen, R. L. (2004) Cauda equina syndrome as a postoperative complication of lumbar spine surgery. Neurosurg. Focus 16(6), e7.

Kang, J. K., Lee, K. S., Jeun, S. S., Lee, I. W., Kim, M. C. (2003) Role of surgery for maintaining urological function and prevention of retethering in the treatment of lipomeningomyelocele: experience recorded in 75 lipomeningomyelocele patients. Childs Nerv. Syst. 19(1), 23-29.

Lapsiwala, S. B., Iskandar, B. J. (2004) The tethered cord syndrome in adults with spina bifida occulta. Neurol. Res. 26(7), 735-740.

Maki, Y., Takayama, M., Hayashi, H., Yokoyama, Y., Agawa, Y. (2017) Cauda equina syndrome due to dural sac shift with engorgement of the epidural venous plexus. Rare complication after lumbar microdiscectomy. World Neurosurg. 104, 1048.e15-1048.e18.

Ramon, S., Domínguez, R., Ramírez, L., Paraira, M., Olona, M., Castello, T., Garcia Fernández, L. (1997) Clinical and magnetic resonance imaging correlation in acute spinal cord injury. Spinal Cord 35(10), 664-673.

Srinivas, S., Shetty, R., Collins, I. (2012) Symptomatic lumbar disc protrusion causing progressive myelopathy in a low-lying cord. Global Spine J. 2(2), 115-118.

Yamada, S., Knerium, D. S., Mandybur, G. M., Schultz, R. L., Yamada, B. S. (2004) Pathophysiology of tethered cord syndrome and other complex factors. Neurol. Res. 26(7), 722-726.

Tabibkhooei A.; Kazemi F.; Kazemi F.; Taheri M. 